\title{
Initial operation of the NSTX phased array for launching high harmonic fast waves
}

\author{
$\underline{\text { P. M. Ryan }}^{\text {a, }}{ }^{*}$, J. R. Wilson ${ }^{b}$, D. W. Swain ${ }^{\mathrm{a}}$, R. I. Pinsker ${ }^{\mathrm{c}}$, M. D. Carter ${ }^{\mathrm{a}}$, D. Gates ${ }^{\mathrm{b}}$,

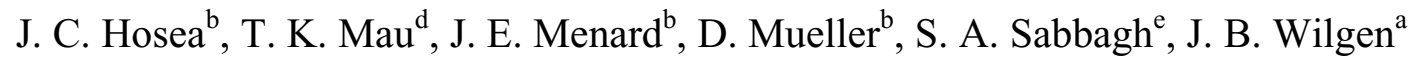 \\ a Oak Ridge National Laboratory, Oak Ridge, Tennessee 37831-8071, USA \\ b Princeton Plasma Physics Laboratory, Princeton, New Jersey, 08543, USA \\ c General Atomics, La Jolla, California, 92121, USA

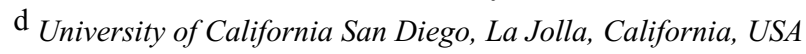 \\ e Columbia University, New York, NY, USA
}

\begin{abstract}
A high harmonic fast wave (HHFW) antenna array, designed to provide up to $6 \mathrm{MW}$ of power at 30 MHz for heating and current drive applications, has been operated on the NSTX experiment at Princeton Plasma Physics Laboratory (PPPL). The full array consists of twelve evenly spaced, identical current strap modules connected in pairs. Each pair is connected as a half-wave resonant loop and is intended to be driven by one transmitter, allowing rapid phase shift between transmitters. A decoupling network compensates for the mutual inductive coupling between adjacent current straps, effectively isolating the six transmitters from one another. Initial rf operation between November 1999 and January 2000 used eight straps to form four loops, which were driven by two transmitters. Two adjacent loops were connected with a $\lambda / 2$ coax section to be driven out of phase by a single transmitter. Up to $2 \mathrm{MW}$ of power was delivered during this stage of operation; inter-loop phasings of $0-\pi-\pi-0$ and $0-\pi-0-\pi$ were investigated. Models of the power distribution system indicate the nominal plasma loading was about $5 \Omega / \mathrm{m}$, close to the design value of $6 \Omega / \mathrm{m}$. The HHFW system has now been reconfigured for 12-strap, 6 -transmitter operation with decouplers; low power vacuum and plasma measurements have begun.
\end{abstract}

Keywords:

* Corresponding author. Tel.: + 1-865-5741133; fax + 1-865-5767926.

E-mail address: ryanpm@ornl.gov (P. M. Ryan) 


\section{Introduction}

The National Spherical Tokamak Experiment (NSTX) is a low aspect ratio tokamak located at Princeton Plasma Physics Laboratory (PPPL), with $R_{0}=0.85 \mathrm{~m}, a \approx 0.67 \mathrm{~m}, \kappa \leq 2, I_{p} \leq 1 \mathrm{MA}, B_{0} \approx 0.25 \mathrm{~T}$. The high harmonic fast wave (HHFW) system, operating at $30 \mathrm{MHz}$, will deliver on the order of $6 \mathrm{MW}$ of auxiliary heating in order to test $\beta$ limits and provide electron heating/current drive for extending the pulse length and demonstrating non-inductive startup. Typical ohmic pulses have a 200 ms duration with average densities in the 0.5 to $1 \times 10^{19} \mathrm{~m}^{-3}$ range. Calculations indicate that most $(\geq 90 \%)$ of the power will be absorbed by the electrons [1]; the HHFW frequency of $30 \mathrm{MHz}$ corresponds to $\sim 16 \omega_{c i}$ for the deuterium ion majority. The antenna array is composed of 12 toroidally-spaced straps, an integral multiple of the six power supplies, in order to improve the launched wave spectrum (higher directionality and narrower peak width) and to reduce the power per strap [2]. The array, covering a toroidal quadrant of the machine, must couple rf power and drive current effectively over a $\beta$ range of 5-40\%, requiring rapid control of the phasing as $\mathrm{T}_{\mathrm{e}}$ changes. At startup, the low $\mathrm{T}_{\mathrm{e}}$ needs a low wave phase velocity corresponding to $90-120^{\circ}$ interstrap phasing for adequate single-pass absorption; the required interstrap phasing is around $30^{\circ}$ for optimum current drive at $\beta=40 \%$.

\section{The HHFW System}

The HHFW system is a 12-element array, fed by six transmitters, which subtends almost a full toroidal quadrant of NSTX. Each of the twelve straps is $0.85 \mathrm{~m}$ long by $0.075 \mathrm{~m}$ wide and is surrounded by a $50 \%$ optically transparent Faraday shield and boron nitride limiters, which can be seen in Fig. 1. The straps are grounded at the bottom and driven at the top through individual vacuum feedthroughs. The characteristic impedance of an isolated strap is about $55 \Omega$ and the phase propagation velocity along the strap is $0.55 \mathrm{c}$. The straps are connected in pairs (as shown in Fig. 2) with a total ground-to-ground electrical length of $2 \lambda$ 
$(20 \mathrm{~m})$; the grounds are configured such that the poloidally-directed strap currents are $180^{\circ}$ out-of-phase. In this arrangement the progressive element phase shift is synchronized between elements 6 and 7 when the transmitter progressive phase shift is either $30^{\circ}$ or $90^{\circ}$. The launched spectrum also has its highest directivity around these two transmitter phasings.

The close spacing of the straps and the transparency of the Faraday shields give rise to large values of inductive coupling between array elements. The coupling coefficient is about 0.1 between neighboring straps and 0.02 between next-to-nearest neighbors, thus requiring a decoupling network to isolate the loops for phase control. Decoupling is accomplished by providing shunt reactance between transmission lines to compensate for the inductive coupling between loops. Coupling between five of the six transmitters occurs through two strap interactions per loop; the coupling between transmitters 1 and 6 occurs through only one strap interaction (straps 6 and 7). Decouplers 1-5 have shunt capacitive reactive provided by 15-300 pF variable vacuum capacitors while decoupler 6 has shunt inductive reactance provided by a shorted stub. Approximately $100 \mathrm{~m}$ of unmatched 9" transmission lines separate the cubes where the resonant loop legs and the decouplers are connected from the line stretchers and stub tuners that provide impedance matching to the transmitters.

The electrical characteristics calculated in the design phase have been checked against measurements on both a full-scale 6-element mockup array [3] and on the installed 12-element array and found to be in good agreement. The system is designed to deliver $6 \mathrm{MW}$ without the peak voltages exceeding $25 \mathrm{kV}$ on the antenna and $35 \mathrm{kV}$ anywhere else in the system, provided the plasma loading is at least $4 \Omega / \mathrm{m}$ [4].

\section{8-strap Array Operation on NSTX}

Only two of the six transmitters were available for NSTX initial operation and were connected to drive eight of the twelve straps (numbers 1,2,3,4,7,8,9,10), as shown in Fig. 3. The eight straps were configured into four resonant loops; the two loops fed by the same transmitter were driven $180^{\circ}$ out-of-phase by $\lambda / 2(5$ m) long connections. The transmitters were operated either in-phase or out-of-phase, giving array phasings of either $0 \pi \pi 0-\pi 00 \pi\left(k_{\text {toroidal }}\right.$ peak at $\left.9 \mathrm{~m}^{-1}\right)$ or $0 \pi 0 \pi-\pi 0 \pi 0\left(k_{\text {toroidal }}\right.$ peak at $\left.13 \mathrm{~m}^{-1}\right)$. The decouplers were not used during this period of operation. 
Changing plasma conditions made it difficult to sustain a sufficiently good match for full power operation during the entire pulse. Up to $2 \mathrm{MW}$ total power was delivered to the plasma briefly; more typical were 1-1.5 MW power levels for 50-100 ms. Significant electron heating was observed when launching waves with lower phase velocity $(0 \pi 0 \pi$ phasing). Figure 4 shows the change in plasma energy (obtained from EFIT analysis of the magnetic equilibrium) caused by the addition of $\sim 1 \mathrm{MW}$ of HHFW power for 50 ms during the current flattop phase. For these shots $I_{p} \approx 550 \mathrm{kA},\left\langle n_{e}>\approx 0.6 \times 10^{19} \mathrm{~m}^{-3}\right.$, and $P_{O H} \approx 1.4 \mathrm{MW}$. The $30 \%$ increase in energy is in agreement with $P^{1 / 2}$ L-mode confinement scaling. Lower electron heating efficiency at higher phase velocities ( $0 \pi \pi 0$ phasing) may be due to H-minority damping, as discussed in $[5]$.

The power reflection coefficient at the transmitters and the positions of the line stretcher and stub tuner to obtain a plasma match were used in conjunction with a coupled transmission line model of the system to determine the plasma resistive loading per unit length of the strap $\left(R^{\prime}\right)$ and any plasma-induced changes in the strap inductance per unit length $\left(L^{\prime}\right)$. Figure 5 shows the time evolution of $R^{\prime}$ and $L^{\prime}$ during a shot that was limited on the inner wall. The $15 \%$ decrease in $L^{\prime}$ from its vacuum value as the gap between the plasma and antenna is reduced is in general agreement with inductance changes calculated if the plasma edge is simulated with a simple conducting boundary. The coupled transmission line analysis which calculates the $L^{\prime}$ from reflection coefficient measurements assumes that the interstrap coupling coefficient is not changed by the plasma but remains constant and symmetric. The asymmetry observed in the plasma loading seen by the two transmitters may be due to the large pitch angle in $\mathbf{B}$ at the antenna; code calculations predict such an effect, but present uncertainties in the edge density profile preclude any definitive statements.

\section{12-strap Array Operation}

During the first half of 2000 , the other four transmitters were commissioned to nominal full power operation at $30 \mathrm{MHz}$, the four remaining antennas were connected into two additional resonant loops, and the decoupling network was configured as in Fig. 2. The quarter-wave transformer sections shown in Fig. 2, intended to lower the VSWR in the unmatched line section, were removed during the eight-strap 
operation and will not be reinstalled unless necessary. Low power measurements $(<5 \mathrm{~W})$ were made during plasma experiments in July 2000 to check overall rf system operation; most of the plasmas were He discharges which were not optimized for rf coupling. The decouplers worked as expected, exhibiting a transmission coefficient between adjacent loops of $-30 \mathrm{~dB}$ in vacuum and $-20 \mathrm{~dB}$ in plasma. The largest interloop coupling now occurs between next-to-nearest neighbors, where the transmission coefficient is a manageable $-10 \mathrm{~dB}$ in the presence of plasma. The transmission lines could be matched independently of each other and each of the six lines used the same relative changes in stub and line stretcher to go from a vacuum match to plasma match, indicating similar plasma loading per loop.

HHFW experiments with the full six-transmitter, twelve-strap array with decouplers are scheduled to commence in September, 2000.

\section{Acknowledgement}

Research sponsored by Office of Fusion Energy Sciences, U.S. Department of Energy, under contract DE-AC05-00OR22725 with Oak Ridge National Laboratory, managed by UT-Battelle, LLC, and under contract DE-AC02-76CH03073 with Princeton Plasma Physics Laboratory. 


\section{References}

[1] M. Ono, High harmonic fast waves in high beta plasmas, Phys. Plasmas 2 (1995) 4075-4082.

[2] J. R. Wilson, et al, High harmonic fast wave heating and current drive on NSTX - system and experimental plan, in S. Bernabei and F. Paoletti (ed.) Radio Frequency Power in Plasmas, $13^{\text {th }}$ Topical Conference, American Institute of Physics, Melville, NY, 1999, pp. 168-171.

[3] P. M. Ryan, et al, Electrical testing of the full-scale model of the NSTX HHFW antenna array, in S. Bernabei and F. Paoletti (ed.) Radio Frequency Power in Plasmas, $13^{\text {th }}$ Topical Conference, American Institute of Physics, Melville, NY, 1999, pp. 453-456.

[4] P. M. Ryan, et al, Design of the HHFW heating and current drive system for NSTX, $2^{\text {nd }}$ Europhysics Topical Conference on Radio Frequency Heating and Current Drive of Fusion Devices, EPS Conf Abs 22A (Brussels, 1998) pp. 97-100.

[5] D. W. Swain, et al, Results of high-harmonic fast wave experiments on NSTX, Proc. 27 ${ }^{\text {th }}$ EPS Conf on Controlled Fusion and Plasma Physics, Budapest, 2000, to appear. 


\section{Figure Captions}

Fig. 1. NSTX HHFW antenna array

Fig. 2. Circuit for 12-strap, 6-transmitter operation with decouplers.

Fig. 3. Circuit for 6-strap, 2-transmitter operation..

Fig. 4. Change in stored energy with $1 \mathrm{MW}$ total HHFW heating.

Fig. 5. Antenna loading and inductance change as the plasma gap is varied during a shot (\#101544). 


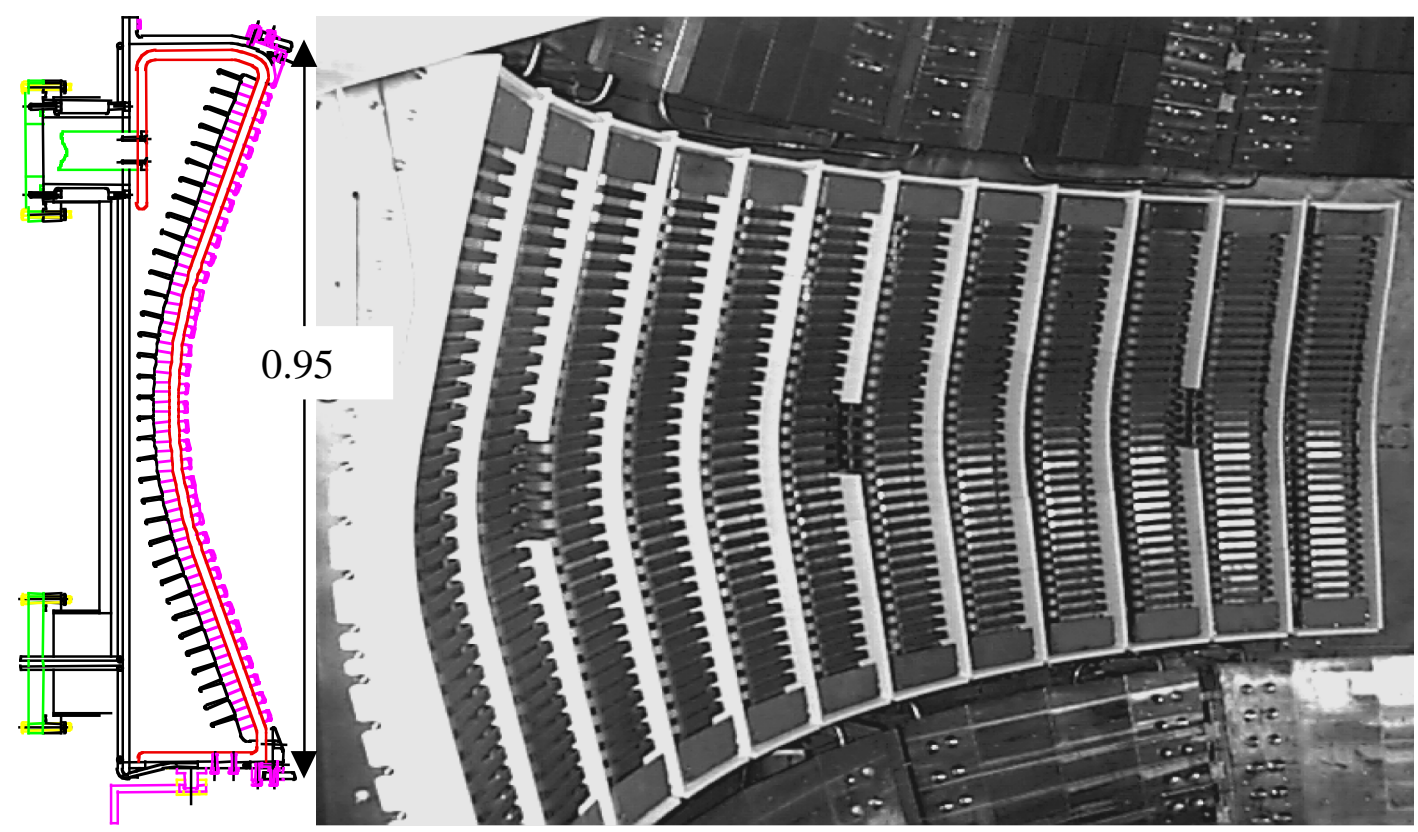




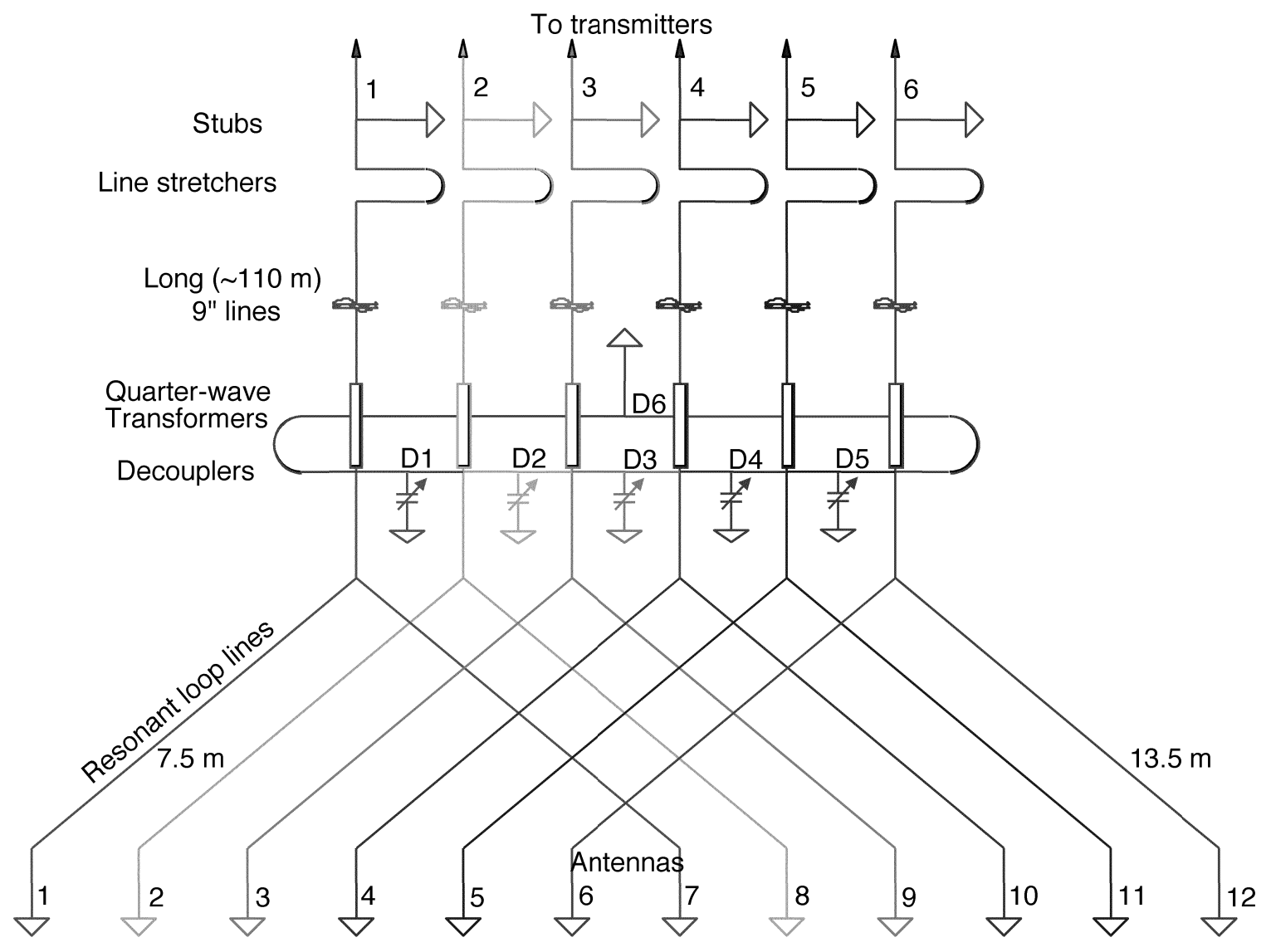




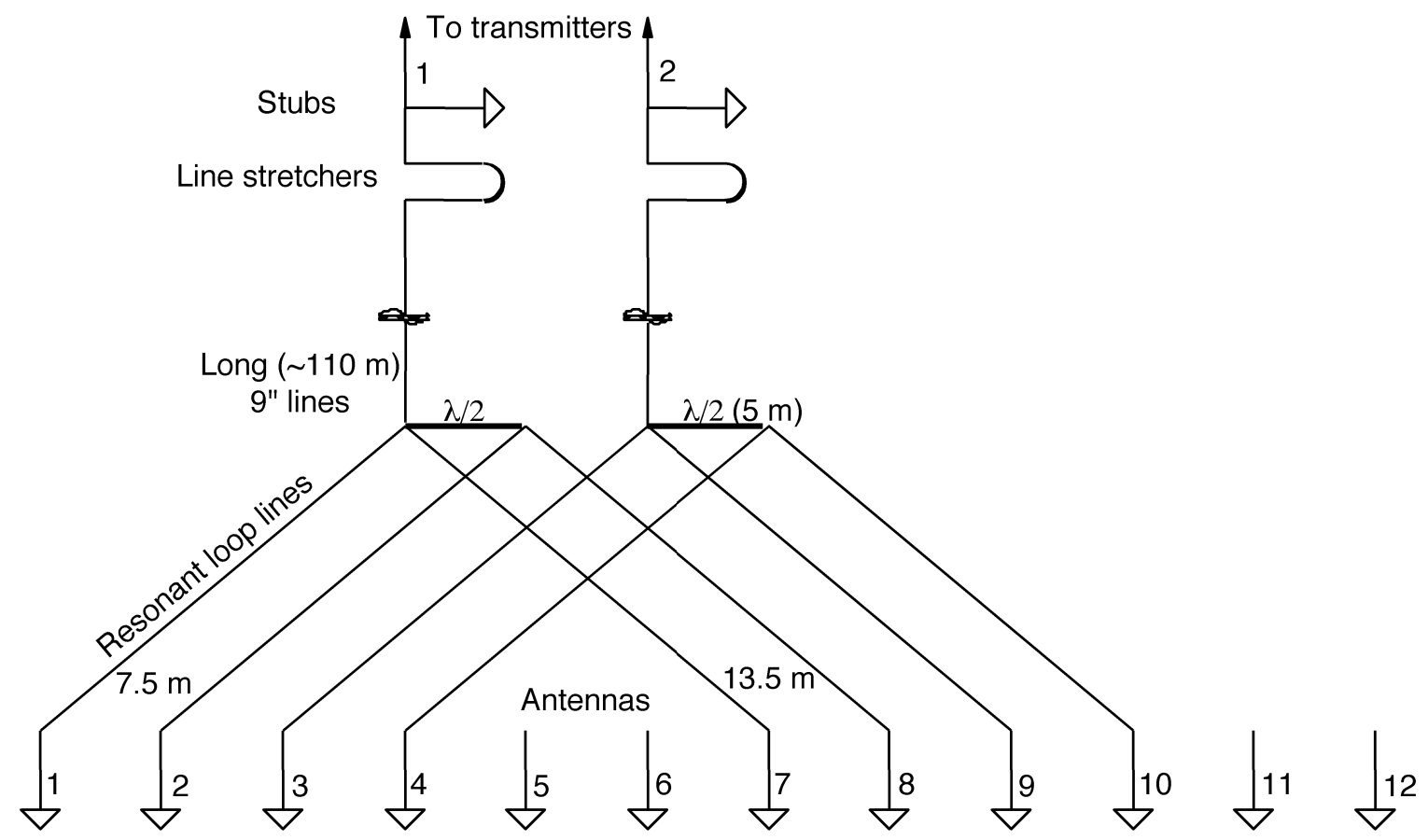




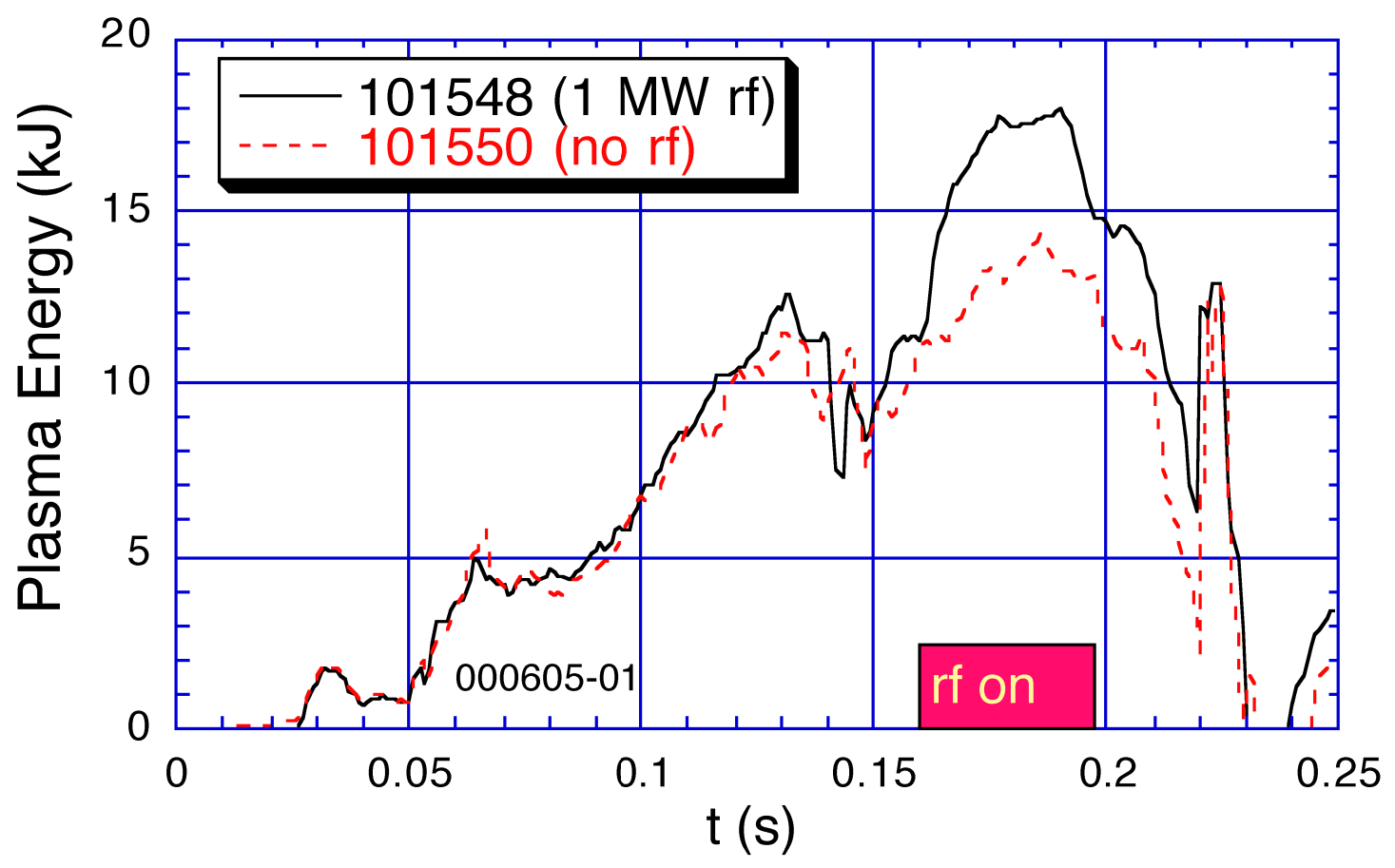



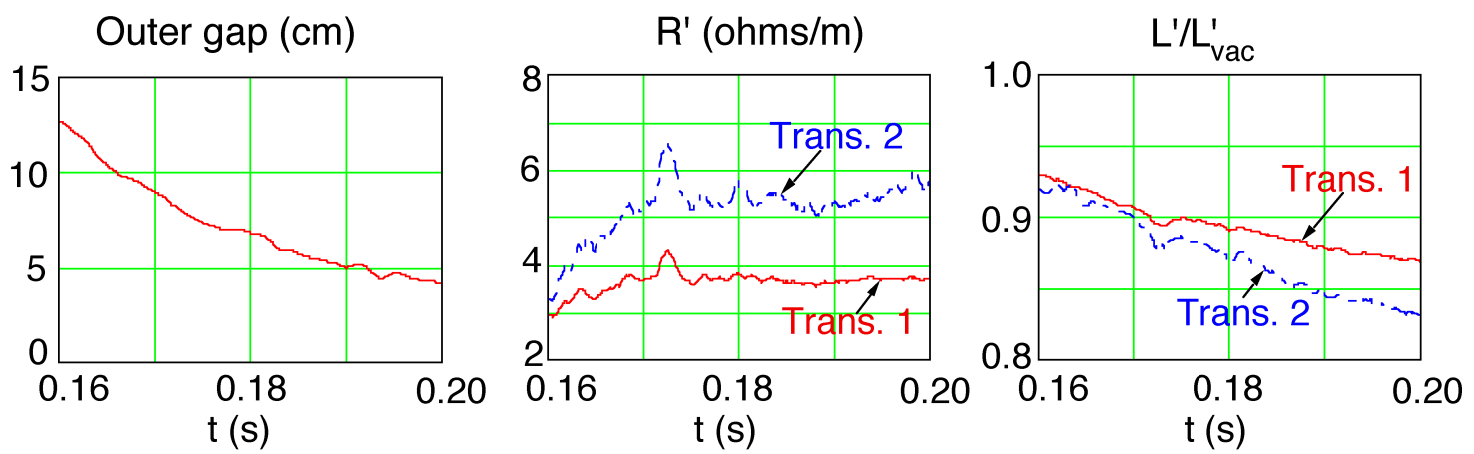